\title{
Preoperative hematological parameters are inadequate for pre- dicting mortality in Stanford Type A aortic dissection repair
}

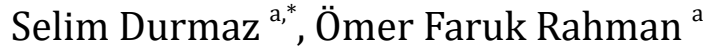 \\ ${ }^{a}$ Department of Cardiovascular Surgery, Faculty of Medicine, Aydın Adnan Menderes University, Aydın, Turkey.
}

\begin{abstract}
Background: Mortality in acute Type A aortic dissection is still high and unpredictable. We aimed to investigate the validity of preoperative hematological markers and possible risk factors in predicting in-hospital mortality in patients operated with deep hypothermic circulatory arrest method.

Methods: 78 consecutive patients who were admitted to the emergency service and operated on were retrospectively analyzed. Risk factors for in-hospital death were investigated to develop a predictive model.

Results: There was no difference between patients in terms of the were demographic data of the patients. In the mortality group, only preoperative creatinine levels were found to be higher $(p<0.05)$. Factors affecting mortality were found as total circulatory arrest (TCA) and cross-clamp (X-clamp) times when intraoperative data were examined $(\mathrm{p}<0.05)$. ROC analysis was performed to determine the power to predict mortality and to determine the cut-off point. In ROC analysis to predict mortality, X-Clamp time $>71$ minutes, $68.2 \%$ sensitivity and $66.1 \%$ specificity, TCA $>44.5$ minutes, $72.7 \%$ sensitivity and $73.2 \%$ specificity were found. In the mortality group, these values were found to be significantly higher than those who were discharged.

Conclusion: In the surgical treatment of Type A aortic dissection under deep hypothermia, hematologic biomarkers may be insufficient in estimating the risk for mortality.

Keywords: Acute; aortic dissection; biomarker; mortality
\end{abstract}

\section{INTRODUCTION}

Acute aortic dissection (AAD) is a life-threatening disease that requires rapid diagnosis and is seen in $0.3 \%$ of patients who present to the emergency department with chest pain ${ }^{[1,2]}$. As a result of the tear between the intima and media layers of the aorta, restricted or progressing along the entire aorta, it may cause symptoms of all organ systems ${ }^{[3,4]}$. Dissections starting from the ascending aorta are classified as Type A, and dissections starting from the distal of the left subclavian

\footnotetext{
* Corresponding author: Selim Durmaz

Mailing address: Department of Cardiovascular Surgery, Faculty of Medicine, Aydın Adnan Menderes University, Aydın 09100, Turkey.

E-mail: sdurmaz@adu.edu.tr

Received: 15 April 2021 / Accepted: 26 May 2021
}

artery are classified as Type B. The current clinical procedure is the reconstruction of Type A AAD (TAAD) with emergency surgical intervention ${ }^{[5]}$. The mortality rate of patients who have raised mortality within hours after diagnosis and who stay without treatment exceeds $90 \%$ within a year. Despite satisfactory results of surgical treatment, it still has high morbidity and mortality rates of up to $40 \%{ }^{[6]}$.

Mortality in AAD is caused by a systemic inflammatory reaction that starts with the dysfunction in multipleorgan perfusion secondary to the mechanical effect created by blood penetrating between the aortic layers ${ }^{[7]}$. Various tests are used in the emergency room to evaluate systemic inflammation. Therefore, the erythrocyte distribution width (RDW), thrombocyte/ lymphocyte ratio (PLR), neutrophil/lymphocyte ratio (NLR), and mean platelet volume (MPV) measurements were obtained from the preoperative whole blood sample have started to be used frequently ${ }^{[8]}$. 
It has also been reported that it can provide predictive information about the prognosis of cardiovascular diseases ${ }^{[9]}$. Although early diagnosis and preoperative variables have positive effects on survival, predicting mortality in TAAD disease is still a question that needs an answer. It would be interesting to develop tests that would quickly assess the severity of surgical operations to be performed on patients. However, determining the risk in surgical interventions may be dependent on preoperative variables as well as intraoperative variables.

Our aim in planning this retrospective clinical study was to determine the effectiveness of preoperative and intraoperative variables to predict mortality in patients with TAAD.

\section{MATERIALS AND METHODS}

\section{Study Population}

A total of 82 consecutive patients who were diagnosed with acute TAAD in the emergency department from January 2010 to January 2018 and operated on urgently were retrospectively analyzed. Patients who underwent deep hypothermic cardiopulmonary arrest but not applied brain protection techniques were included in the study. After the study protocol was approved by Aydın Adnan Menderes University Non-Interventional Clinical Studies Ethics Committee (Ref: 53043469 050.04.04), the data were retrospectively prepared and recorded. Patients with known hematological diseases, cancer, immunological diseases, and infections were excluded from the study. Hematological and biochemical values of 78 patients included in the study at the time of admission to the emergency department were recorded. Operational data were obtained by examining the surgery reports in files that are stored in electronic media.

\section{Operative Technique}

Deep hypothermic $\left(18{ }^{\circ} \mathrm{C}\right)$ cardiopulmonary bypass was performed in all patients under general anesthesia via standard aortic, femoral, or axillary arterial cannulation and femoral or right atrial venous cannulation. Antegrade hypothermic and hyperkalemic blood cardioplegia was performed in all patients. When the deep hypothermic circulatory arrest (DHCA) was achieved, sodium pentothal was administered. The intimal tear area was found with aortotomy and was excised and made suitable for anastomosis. The anastomosis was conducted using the Dacron vascular graft instead of the removed ascending aorta. After the anastomoses were finished, the patient was started to be warmed, cardiopulmonary bypass was terminated, and the patient was transferred to intensive care.

\section{Study groups}

Study groups were divided according to the Stanford aortic dissection classification. Among the patients with TAAD, those with early postoperative mortality (within the first 30 days) were classified as Exitus, and the patients who were discharged were classified as Discharge. Regardless of hunger, preoperative blood samples taken in the emergency room were taken. Using Mindray BC 6800 (Mindray, China), full blood counts were collected, including total white blood cells, neutrophils, lymphocytes, and platelets. The ratio of thrombocytes $\left(10^{3} / \mathrm{uL}\right)$ to lymphocytes $\left(10^{3} / \mathrm{uL}\right)$ collected from blood samples was calculated as PLR, and the ratio of neutrophils $\left(10^{3} / \mathrm{uL}\right)$ to lymphocytes $\left(10^{3} /\right.$ uL) was calculated as NLR.

\section{Statistical analysis}

For statistical analysis, the data were analyzed using the Statistical Package for the Social Sciences (SPSS) program (IBM SPSS Statistics for Windows, Version 18.0. Armonk, NY, USA). The study of normality was carried out using either the Kolmogorov-Smirnov or Shapiro-Wilk measures. Chi-square test was used for categorical variables and Mann Whitney u test was used for continuous variables. Receiver operating curve (ROC) analysis was performed to determine the factors that may affect mortality. The significance level was accepted as $\mathrm{p}<0.05$ for all statistical evaluations.

Table 1. Demographic characteristics of patients

\begin{tabular}{llll}
\hline & Discharge & Exitus & p-value \\
\hline Age (Years, Mean \pm SD) & $59,28 \pm 11,39$ & $57,13 \pm 17,19$ & $0,276 \#$ \\
BMI(kg/m2, Mean \pm SD) & $28,08 \pm 4,6$ & $28,36 \pm 4,38$ & $0,803 \#$ \\
EF (\%, Mean \pm SD) & $49,66 \pm 10,08$ & $51 \pm 9,46$ & $0,536 \#$ \\
Female (n) & 10 & 6 & $0,365^{*}$ \\
Male (n) & 46 & 16 & $0.264^{*}$ \\
HT (n) & 32 & 14 & 0,600 \\
DM (n) & 25 & 12 & 0,431 \\
COPD (n) & 9 & 3 & $0,546^{*}$ \\
DL (n) & 15 & 5 & 0,712 \\
Family History (n) & 2 & 0 & $0,513^{*}$ \\
Smoking (n) & 28 & 10 & 0,718 \\
\hline
\end{tabular}

BMI: Body mass index, EF: Ejection fraction, HT: Hypertension, DM: Diabetes Mellitus, COPD: Chronic Obstructive Pulmonary Disease, DL: Dyslipidaemia. *Chi-square, \#Mann Whitney-U test.

\section{RESULTS}

The demographic data of the patients are shown in Table 1. No difference was observed between discharged 
Table 2. Characteristics of blood values of patients

\begin{tabular}{llll}
\hline & Discharge & Exitus & p-value* \\
\hline Hemoglobin & $13.20 \pm 1.61$ & $13.63 \pm 1.48$ & 0.257 \\
Htc & $39.44 \pm 4.27$ & $40.81 \pm 4.17$ & 0.196 \\
Neutrophils, $10^{3} / \mathrm{uL}$ & $6.13 \pm 4.48$ & $5.85 \pm 1.84$ & 0.564 \\
Lymphocytes, $10^{3} / \mathrm{uL}$ & $2.06 \pm 0.89$ & $2.14 \pm 0.86$ & 0.586 \\
Platelets, $/ \mathrm{mm}^{3}$ & $278.28 \pm 88.53$ & $288.45 \pm 66.85$ & 0.328 \\
MPV & $9.73 \pm 7.64$ & $8.51 \pm 1.02$ & 0.515 \\
RDW & $16.44 \pm 14.67$ & $13.94 \pm 1.16$ & 0.153 \\
BUN, mg/dl & $38.55 \pm 19.52$ & $36.72 \pm 12.24$ & 0.894 \\
Creatinine, mg/dl & $0.82 \pm 0.20$ & $0.97 \pm 0.34$ & 0.041 \\
PLR & $163.30 \pm 92.21$ & $158.18 \pm 81.45$ & 0.903 \\
NLR & $3.49 \pm 3.30$ & $3.19 \pm 1.81$ & 0.798 \\
CK-MB, mg/dl & $99.73 \pm 54.45$ & $118.82 \pm 51.20$ & 0.162 \\
LDH, mg/dl & $98.02 \pm 29.86$ & $99.05 \pm 31.45$ & 0.903 \\
AST, mg/dl & $78.29 \pm 41.56$ & $74 \pm 41.32$ & 0.673 \\
ALT, mg/dl & $39.38 \pm 18.43$ & $42.68 \pm 15.85$ & 0.477 \\
\hline
\end{tabular}

Htc: Hematocrit, MPV: Mean platelet volume, PDW: Platelet distribution width, RDW: Red blood cell distribution width, BUN: Blood urea nitrogen, PLR: Platelet to lymphocyte ratio, NLR: Neutrophil to lymphocyte ratio, CK-MB: Creatinine kinase myocardial band, LDH: Lactate dehydrogenase, AST: Aspartate transaminase, ALT: Alanine transaminase. *Mann Whitney-U test.

patients and patients who died. When the data were examined in terms of preoperative blood values, there was no difference in blood parameters such as Haemoglobin (Hb), PLR, NLR, RDW, MPV, ALT, AST, LDH, and CK-MB. Only preoperative creatinine levels were found to be higher in the mortality group $(\mathrm{p}<0.05)$ (Table 2$)$. The display of intraoperative data is given in Table 3. When these data are examined, the effect of cardiopulmonary bypass time on mortality has not been found. It was found that the factors affecting mortality were total circulatory arrest (TCA) and Cross clamp (X-clamp) times $(\mathrm{p}<0.05)$. In univariate analysis, creatinine (odds ratio [OR]: 9.26; 95\% CI: 1.304-65.84, $\mathrm{p}=0.026$ ), X-clamp (OR: 1.048; 95\% CI: 1.016-1.082, $\mathrm{p}=0.003$ ) and TCA (OR: 1.109; 95\% CI: 1.047-1.174, $\mathrm{p}=0.000$ ) were associated with in-hospital mortality after TAAD surgery. In the multivariate logistic regression model with the backward elimination method, $\mathrm{X}$ clamp (OR: 1.031; 95\% CI: 1.003-1.060, p < 0.032), and TCA (OR: 1.089; 95\% CI: 1.025-1.157, $\mathrm{p}=0.006$ ) remained as independent predictors of in-hospital mortality after TAAD surgery (Table 4).

Table 3. Intraoperative characteristics of patients

\begin{tabular}{llll}
\hline & Discharge & Exitus & p-value* \\
\hline X-Clamp (m, Mean \pm SD) & $65.53 \pm 17.57$ & $84.68 \pm 25.02$ & 0.00 \\
TCA $(m$, Mean \pm SD) & $39.76 \pm 9.78$ & $50.63 \pm 10.34$ & 0.00 \\
CPB $(m$, Mean \pm SD) & $132.73 \pm 29.13$ & $144.40 \pm 38.24$ & 0.03 \\
\hline
\end{tabular}

X-Clamp: Cross clamp time, TCA: Total circulatory arrest time, CPB: Cardiopulmonary bypass time (minute), *Mann Whitney-U test.
Table 4. Results of univariate and multivariate analysis of predictors of in-hospital mortality

\begin{tabular}{lcclc}
\hline \multirow{2}{*}{ Variable } & \multicolumn{2}{c}{ Univariate } & \multicolumn{2}{c}{ Multivariate } \\
\cline { 2 - 5 } & $\begin{array}{c}\text { Odds ratio } \\
\text { (95\% CI) }\end{array}$ & p-value & $\begin{array}{c}\text { Odds ratio } \\
\text { (95\% CI) }\end{array}$ & p-value \\
\hline Creatinine & $9.26(1.304-65.84)$ & 0.026 & $4(0.444-36.065)$ & 0.217 \\
X-Clamp & $1.048(1.016-1.082)$ & 0.003 & $1.031(1.003-1.060)$ & 0.032 \\
TCA & $1.109(1.047-1.174)$ & 0.000 & $1.089(1.025-1.157)$ & 0.006 \\
\hline
\end{tabular}

The receiver operating characteristics curve was used to determine the sensitivity and specificity of TCA and $\mathrm{X}$-clamp time and the optimal cut-off value for predicting mortality (Figure 1). In ROC analysis, X-Clamp time $>71 \mathrm{~m}$. predicted mortality with $68.2 \%$ sensitivity and $66.1 \%$ specificity (AUC: $0.788, \mathrm{p}<0.05$ ), TCA $>44.5 \mathrm{~m}$. predicted mortality with $72.7 \%$ sensitivity and $73.2 \%$ specificity (AUC: $0.778, \mathrm{p}<0.05$ ). It was found that these times were significantly higher in the mortality group than those who were discharged.

\section{DISCUSSION}

This study examined the relationship between mortality and preoperative hematological markers in patients who underwent surgery for TAAD. The results we found in our study determined that preoperative hematological markers failed to predict mortality. However, it showed that intraoperative variables, X-clamp time and TCA times, are more important in predicting mortality.

TAAD is a vascular clinical pathology that occurs with high morbidity and mortality, and its incidence is increasing with prolongation of life span and prevalence of hypertension. Patients and doctors are still concerned about the high risk of surgery. In previous studies, the efficacy of hematological markers, a simple and inexpensive method, predicting thrombosis, inflammation, and mortality was investigated. Most of the studies conducted to show mortality are related to Type B $\mathrm{AAD}^{[10]}$. The time from diagnosis to surgery may allow such assessments in patients diagnosed with Type B AAD. However, this option can often be overlooked due to the need for rapid surgery decisions in patients diagnosed with TAAD.

Several recent studies have found an association between increased hematological inflammatory markers and mortality ${ }^{[11,12]}$. Sbarouni et al. stated that PLR and NLR could be used for diagnostic purposes in a group of patients with TAAD ${ }^{[13]}$. In another study stating that preoperative hematological markers have a predictive effect on mortality, Erdolu et al. reported that CRP 


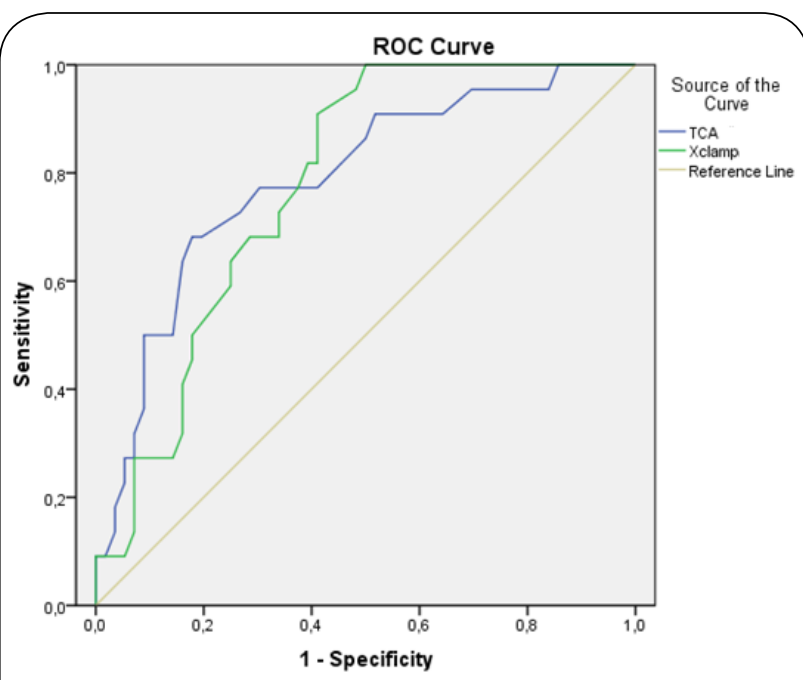

Figure 1. Receiver operating curve characteristics of intraoperative variables to predict mortality in the study groups. TCA: Total circulatory arrest time, X-clamp: Cross clamp.

and NLR were predictors of mortality ${ }^{[14]}$. In our study, we could not find the predictive value of these markers. However, when these studies and our studies are examined, it is controversial that it predicts mortality despite its diagnostic benefit. One reason why it could not be detected as a mortality indicator in our study may be that the preoperative inflammation level may not be high enough to cause mortality. Considering the inflammation caused by intraoperative reasons, it may be insufficient to determine mortality. In addition, surgical technique differences may influence in-hospital mortality. The use of the deep hypothermic circulatory arrest technique in our study may have had a severe effect on intraoperative inflammation.

Many tests have been used to predict mortality. However, the reliability of these tests was limited due to a major surgical operation affecting mortality. It was stated that the factors affecting mortality were age, preoperative renal dysfunction, and prolongation of CPB duration ${ }^{[15-18]}$. In the international registry of acute aortic dissection (IRAD) study, Rampoldi et al. ${ }^{[19]}$, it was found that prolongation of intraoperative TCA duration was associated with mortality. In our study, it was found to be associated with prolonged X-clamp and TCA durations. Graft anastomosis time, which is one of the factors that may affect the prolongation of TCA, may be related to intact aortic tissue. In addition, in cases where the dissection line includes the aortic arch, mortality may increase in this patient group as the duration of the anastomosis may increase, resulting in longer X-clamp and TCA times.

Many techniques are used to reduce mortality in the challenging surgical treatment of TAADs. Although DHCA is one of these techniques, it has been shown in recent years that reducing body temperature to moderate hypothermia level with techniques in which antegrade cerebral perfusion is combined reduces mortality. The time to DHCA formation and re-normothermic state may have contributed to mortality by prolonging $\mathrm{CPB}$ time and total operative time. Wen et al. also found that non-TAAD survivors had a much longer X-clamp time than survivors ${ }^{[15]}$. In this study, they stated that improving intraoperative management and shortening the X-clamp time would contribute to improving in-hospital mortality. In addition to shortening the intraoperative time, avoiding a deep hypothermic state may contribute to the reduction of the systemic inflammatory response in the patient and thus to better results.

The main technical difficulty of TAAD operations is that they require a long time and operations are performed under cardiopulmonary bypass. Similarly, we found a generally known relationship between long operative time and in-hospital mortality. However, these periods differ in various studies. X-clamp time Nissinen et al. is $150 \mathrm{~min}$, Kawahito et al. $240 \mathrm{~min}, \mathrm{Wu}$ et al. found that it was associated with the risk of mortality at $160 \mathrm{~min}$ ${ }^{[20-22]}$. In our study, the X-clamp time was found to be 71 minutes according to Roc curve analysis (OR: 1.031; 95\% CI: 1.003-1.060, p < 0.032). Unlike our study, we see that antegrade cerebral perfusion techniques and moderate hypothermia techniques are used in these studies. In TAAD, shortening the intraoperative time and using cerebral protection techniques contribute to safe operation time. Increasing the safe surgical time in operations to be performed under deep hypothermia is an important way to improve in-hospital mortality.

The data used in this study, which was planned as a retrospective cohort study, have limitations. The time from the symptoms of the patients to the hospital admission may affect the level of inflammation. The time that passes due to the distance of the patients from our center may have an impact on mortality. It will be useful to evaluate the results under these conditions. Causes such as late diagnosis, tamponade due to aortic rupture, and multiple organ failure, which are among the data of preoperative conditions that may affect mortality, could not be presented in our study.

In conclusion, TAAD continues to be an emergency cardiovascular pathology with high mortality and challenging treatment. Although studies have been carried out for years to predict mortality, and an effective op- 
tion has not yet been put into use. There is a need for studies involving larger patient groups in which the mortality of patients undergoing surgery is evaluated in addition to preoperative variables, perioperative surgical, and hematologic variables.

\section{DECLARATIONS}

\section{Authors' contributions}

Selim Durmaz: Conceived the project, carried out to acquisition and analysis of data, contributed to drafting and approval of the final manuscript.

Ömer Faruk Rahman: Conceived the project, carried out to acquisition and analysis of data, contributed to drafting and approval of the final manuscript.

\section{Conflict of interest}

All authors declared that there are no conflicts of interest.

\section{REFERENCES}

1. Ohle, R., Um, J., Anjum, O., Bleeker, H., Luo, L., Wells, G., \& Perry, J. J. (2018). High risk clinical features for acute aortic dissection: a case-control study. Academic Emergency Medicine, 25(4), 378-387.

2. Manea, M. M., Dragos, D., Antonescu, F., Sirbu, A. G., Tiron, A. T., Dobri, A. M., \& Tuta, S. (2019). Aortic Dissection: An Easily Missed Diagnosis when Pain Doesn't Hold the Stage. The American journal of case reports, 20, 1788.

3. Mussa, F. F., Horton, J. D., Moridzadeh, R., Nicholson, J., Trimarchi, S., \& Eagle, K. A. (2016). Acute aortic dissection and intramural hematoma: a systematic review. Jama, 316(7), 754-763.

4. Minegishi, S., Watanabe, H., Horita, N., Shibata, Y., Kaneko, T., \& Ishigami, T. (2016). The current evidence on diagnosis and treatment of acute aortic syndrome. Journal of thoracic disease, 8(12), E1617.

5. Baliyan, V., Parakh, A., Prabhakar, A. M., \& Hedgire, S. (2018). Acute aortic syndromes and aortic emergencies. Cardiovascular diagnosis and therapy, 8(Suppl 1), S82.

6. Bashir, M., Harky, A., Fok, M., Shaw, M., Hickey, G. L., Grant, S. W., ... \& Oo, A. (2017). Acute type A aortic dissection in the United Kingdom: surgeon volume-outcome relation. The Journal of thoracic and cardiovascular surgery, 154(2), 398-406.

7. Ramanath, V. S., Oh, J. K., Sundt III, T. M., \& Eagle, K. A. (2009, May). Acute aortic syndromes and thoracic aortic aneurysm. In Mayo Clinic Proceedings (Vol. 84, No. 5, pp. 465-481). Elsevier.

8. Bedel, C., \& Selvi, F. (2019). Association of platelet to lymphocyte and neutrophil to lymphocyte ratios with in-hos- pital mortality in patients with type A acute aortic dissection. Brazilian journal of cardiovascular surgery, 34(6), 694-698.

9. Monteiro Júnior, J. G. D. M., \& de Oliveira Cipriano Torres, D. (2019). Hematological parameters as prognostic biomarkers in patients with cardiovascular diseases. Current Cardiology Reviews, 15(4), 274-282.

10. Zhang, J., Cheng, B., Yang, M., Pan, J., Feng, J., \& Cheng, Z. (2019). Predicting in-hospital death in patients with type B acute aortic dissection. Medicine, 98(32).

11. Liu, H., Li, D., Jia, Y., \& Zeng, R. (2020). Predictive Value of White Blood Cells, Neutrophils, Platelets, Platelet to Lymphocyte and Neutrophil to Lymphocyte Ratios in Patients with Acute Aortic Dissection. Brazilian Journal of Cardiovascular Surgery, 35(6):1031-1033..

12. Li, D. Z., Chen, Q. J., Sun, H. P., Zeng, R., Zeng, Z., Gao, X. M., ... \& Yang, Y. N. (2016). Mean platelet volume to platelet count ratio predicts in-hospital complications and longterm mortality in type A acute aortic dissection. Blood Coagulation \& Fibrinolysis, 27(6), 653-659.

13. Sbarouni, E., Georgiadou, P., Kosmas, E., Analitis, A., \& Voudris, V. (2018). Platelet to lymphocyte ratio in acute aortic dissection. Journal of clinical laboratory analysis, 32(7), e22447.

14. Erdolu, B., \& As, A. K. (2020). C-Reactive Protein and Neutrophil to Lymphocyte Ratio Values in Predicting Inhospital Death in Patients with Stanford Type A Acute Aortic Dissection. The Heart Surgery Forum, 23(4), 488-492.

15. Wen, M., Han, Y., Ye, J., Cai, G., Zeng, W., Liu, X., ... \& Zeng, H. (2019). Peri-operative risk factors for in-hospital mortality in acute type A aortic dissection. Journal of thoracic disease, 11(9), 3887.

16. Cabasa, A., \& Pochettino, A. (2016). Surgical management and outcomes of type A dissection-the Mayo Clinic experience. Annals of cardiothoracic surgery, 5(4), 296.

17. Harky, A., Singh, V. P., Khan, D., Sajid, M. M., Kermali, M., \& Othman, A. (2020). Factors Affecting Outcomes in Acute Type A Aortic Dissection: A Systematic Review. Heart, Lung and Circulation, 29(11), 1668-1681.

18. Zhou, W., Wang, G., Liu, Y., Tao, Y., Du, Z., Tang, Y., ... \& $\mathrm{Xu}, \mathrm{Z}$. (2019). Outcomes and risk factors of postoperative hepatic dysfunction in patients undergoing acute type A aortic dissection surgery. Journal of thoracic disease, 11(8), 3225.

19. Rampoldi, V., Trimarchi, S., Eagle, K. A., Nienaber, C. A., Oh, J. K., Bossone, E., ... \& International Registry of Acute Aortic Dissection (IRAD) Investigators. (2007). Simple risk models to predict surgical mortality in acute type A aortic dissection: the International Registry of Acute Aortic Dissection score. The Annals of Thoracic Surgery, 83(1), 55-61.

20. Nissinen, J., Biancari, F., Wistbacka, J. O., Peltola, T., Loponen, P., Tarkiainen, P., ... \& Tarkka, M. (2009). Safe time 
limits of aortic cross-clamping and cardiopulmonary bypass in adult cardiac surgery. Perfusion, 24(5), 297-305.

21. Kawahito, K., Adachi, H., Yamaguchi, A., \& Ino, T. (2001). Preoperative risk factors for hospital mortality in acute type A aortic dissection. The Annals of thoracic surgery, 71(4), 1239-1243.
22. Wu, Y., Jiang, R., Xu, P., Wang, G., Wang, J., \& Yang, S. (2018). Perioperative Results and Risk Factors for In-Hospital Mortality In Patients With Stanford Type A Aortic Dissection Undergoing Sun's Procedure-A Single Center Study. The heart surgery forum, 21(6), 432-437. 